\title{
Erratum: Square-root higher-order topological insulator on a decorated honeycomb lattice [Phys. Rev. A 102, 033527 (2020)]
}

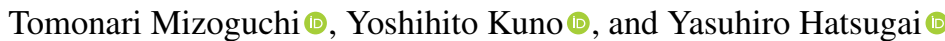

(Received 3 August 2021; published 23 August 2021)

DOI: 10.1103/PhysRevA.104.029906

We found an error in our numerical code, which led to the incorrect value of $p_{\mathrm{H}}$ in Fig. 4 of the original paper. The revised figure is shown below, where we see $p_{\mathrm{H}}$ is $1 / 3$ for $t_{1} / t_{2}<1$ and 0 for $t_{1} / t_{2}>1$. We additionally note that the vertical axis should be $p$ rather than $p / 2 \pi$.

Therefore, the following part in Sec. III of the original paper has to be amended. "We see that $p$ changes from $\frac{1}{3}\left(t_{1} / t_{2}<1\right)$ to $0\left(t_{1} / t_{2}>1\right)$ for $h_{k}^{(\mathrm{K})}$, whereas it is 0 for $h_{k}^{(\mathrm{H})}$. This indicates that the higher-order topology of the present model is inherited from the breathing kagome-lattice model. However, as we have pointed out, the corner modes have amplitudes on both honeycomb (white) and kagome (black) sites, meaning that the actual corner states are not described by the kagome lattice alone. One can also find that the following relation of the topological invariants between the original model and the squared model holds:

$$
2 \pi p_{1} \equiv 2 \pi p_{5} \equiv 2 \pi\left(p_{\mathrm{K}}+p_{\mathrm{H}}\right)(\bmod 2 \pi),
$$

which follows from Eqs. (13) and (15)."

In the corrected figure, we see the following relation holds, instead of Eq. (20) of the original paper:

$$
2 \pi p_{1} \equiv 2 \pi p_{5} \equiv 2 \pi p_{\mathrm{K}} \equiv 2 \pi p_{\mathrm{H}}(\bmod 2 \pi) .
$$

This relation can be derived from Eqs. (6), (13), and (15) of the original paper. To show this, we note the following: (i) The $C_{3}$ rotation operator $U_{\boldsymbol{k}}$ is block diagonalized into the honeycomb and kagome sectors [Eq. (6) in the original paper]. (ii) $\boldsymbol{u}_{1 / 5}(\boldsymbol{k}=K)$ is the eigenvector of $U_{\boldsymbol{k}=K}$ whose eigenvalue is $\theta_{1 / 5}(K)$. Note that the eigenvector $u_{1 / 5}(\boldsymbol{k}=K)$ is composed of $\sqrt{E_{K}^{+}} u^{(\mathrm{H})}(K)$ and $u^{(\mathrm{K})}(K)$ [Eqs. (13) and (15) of the original paper]. Hereafter, we focus on the case of $n=5$, but the same argument holds for $n=1$. On one hand, from (i), we have

$$
\begin{aligned}
U_{K} \boldsymbol{u}_{5}(K) & =\frac{1}{\mathcal{N}_{\boldsymbol{k}}}\left(\begin{array}{cc}
U_{K}^{(\mathrm{H})} & \mathcal{O}_{2 \times 3} \\
\mathcal{O}_{3 \times 2} & U_{K}^{(\mathrm{K})}
\end{array}\right)\left(\begin{array}{c}
\sqrt{E_{K}^{+}} \boldsymbol{u}^{(\mathrm{H})}(K) \\
\boldsymbol{u}^{(\mathrm{K})}(K)
\end{array}\right) \\
& =\frac{1}{\mathcal{N}_{\boldsymbol{k}}}\left(\begin{array}{c}
\sqrt{E_{K}^{+}} U_{K}^{(\mathrm{H})} \boldsymbol{u}^{(\mathrm{H})}(K) \\
U_{K}^{(\mathrm{K})} \boldsymbol{u}^{(\mathrm{K})}(K)
\end{array}\right),
\end{aligned}
$$

where

$$
U_{\boldsymbol{k}}^{(\mathrm{H})}=\left(\begin{array}{cc}
1 & 0 \\
0 & e^{-i \boldsymbol{k} \cdot \boldsymbol{a}_{2}}
\end{array}\right)
$$

and

$$
U_{k}^{(\mathrm{K})}=\left(\begin{array}{lll}
0 & 0 & 1 \\
1 & 0 & 0 \\
0 & 1 & 0
\end{array}\right)
$$

On the other hand, from (ii), we have

$$
U_{K} \boldsymbol{u}_{5}(K)=\theta_{5}(K) \frac{1}{\mathcal{N}_{\boldsymbol{k}}}\left(\begin{array}{c}
\sqrt{E_{K}^{+}} \boldsymbol{u}^{(\mathrm{H})}(K) \\
\boldsymbol{u}^{(\mathrm{K})}(K)
\end{array}\right)
$$

Comparing Eq. (2) with Eq. (5), we find

$$
U_{K}^{(\mathrm{H})} \boldsymbol{u}^{(\mathrm{H})}(K)=\theta_{5}(K) \boldsymbol{u}^{(\mathrm{H})}(K)
$$




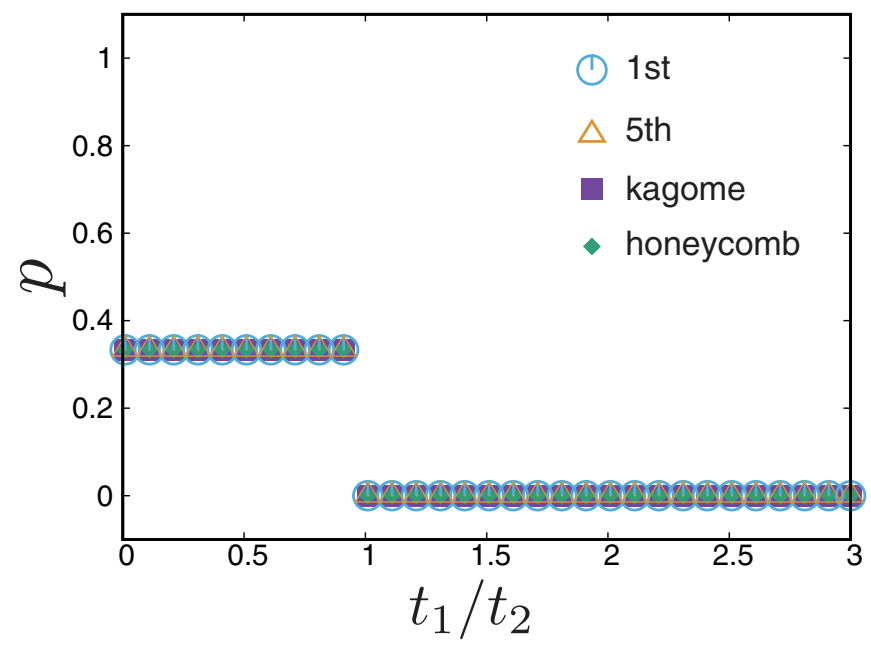

FIG. 4. Polarization of Eq. (18) as a function of $t_{1} / t_{2}$. Blue circles and orange triangles are for the first and the fifth bands of the decorated honeycomb-lattice model, respectively. Purple squares are for the upper dispersive band of the breaking kagome-lattice model $h_{k}^{(\mathrm{K})}$ and the green diamonds are for the upper dispersive band of the honeycomb-lattice model $h_{k}^{(\mathrm{H})}$.

and

$$
U_{K}^{(\mathrm{K})} \boldsymbol{u}^{(\mathrm{K})}(K)=\theta_{5}(K) \boldsymbol{u}^{(\mathrm{K})}(K)
$$

which lead to the relation of Eq. (1).

We note that the main claim in the original paper, i.e., the realization of the square-root higher-order topological insulator on a decorated honeycomb lattice, is not affected by the above correction, because the results regarding the decorated honeycomb lattice are unchanged.

We thank Daiki Matsumoto for drawing our attention to this issue. 